\title{
Characterization and Application of Innovative Plasmonic Arrays
}

\author{
Dana Cialla $^{1,2}$, Karina Weber ${ }^{1,2}$, Uwe Hübner ${ }^{2}$, Henrik Schneidewind ${ }^{2}$, Matthias Zeisberger ${ }^{2}$, Roland \\ Mattheis ${ }^{2}$, Jürgen Popp ${ }^{1,2}$ \\ ${ }^{1}$ Institute of Physical Chemistry and Abbe Center of Photonics, Friedrich-Schiller-University Jena, \\ Helmholtzweg 4, 07743 Jena, Germany, \\ dana.cialla@uni-jena.de; juergen.popp@uni-jena.de \\ ${ }^{2}$ Institute of Photonic Technology (IPHT) Jena, Albert-Einstein-Straße 9, 07745 Jena, Germany
}

\begin{abstract}
:
In order to increase the inherent weak but molecular specific Raman signature the exceptional optical properties of plasmonic nanostructures are used. This technique is termed as surface enhanced Raman spectroscopy (SERS) and combines the fingerprint specificity with potential single molecule sensitivity. However, the non-application of SERS in routine analytics is often linked with the low reproducibility of metallic nanostructures or metallic surfaces. Within this contribution, the fabrication of innovative plasmonic arrays with homogenous signal enhancement is introduced, which is an important contribution towards powerful SERS diagnostics. Here, the application of plasmonic arrays for DNA sensing is demonstrated.
\end{abstract}

Key words: surface enhanced Raman spectroscopy (SERS), plasmonic array, gold nanostructures, DNA sensing

\section{Introduction}

The exceptional optical properties of silver and gold nanostructured particles have been used for artworks and colored glass since the Late Antiquity. Due to the interaction of light with metallic nanoparticles surface plasmon polariton modes are excited. A strong electromagnetic field is induced on the metal surface, which is used in surface enhanced Raman spectroscopy (SERS) for a dramatic amplification of the inherent weak but molecular specific Raman signature [1]. Thus, the SERS technique combines the fingerprint specificity of Raman spectroscopy with an increased sensitivity due to an enhancement of the Raman intensity by several orders of magnitude. However, the SERS technique is not accepted as routine analytical tool, which is often linked with difficulties in the preparation of SERS substrates with homogenous and reproducible signal enhancement [2]. Within this contribution, regular patterned nanostructures providing homogenous signal enhancement are introduced and the application as DNA sensor is shown.

\section{Innovative plasmonic arrays}

To fabricate regular patterned plasmonic arrays with homogenous signal enhancement, electron beam lithography $(E B L)$ is used $[3,4]$. In Fig. 1 the SEM image of a gold nanorhomb array is depicted. The optical parameters are tunable by varying both the size of the nanoparticles and the periodicity of the array [5], which leads to design and fabrication strategies of SERS arrays developed to gain a maximum SERS enhancement [6]. Further, silver is established as plasmonic material in EBL processes. Here, a quartz surface is structured applying EBL and gets coated with silver in a final procedure step [7].

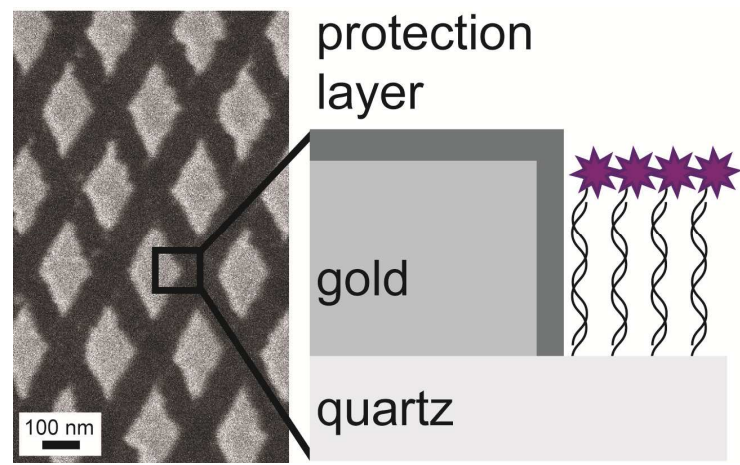

Fig. 1. The SEM image illustrates the periodically patterned gold surface (left). The application scheme for the detection of DNA is shown (right).

Further, enzyme-induced silver nanostructures allowing the fabrication of low-cost and largescale reproducible substrates are applied as 
SERS substrate. The relationship between electrical conductivity and the resulting SERS activity is used to pre-characterize the plasmonic array prior the SERS measurement. Thus, the prediction of the SERS activity of the nanostructure ensemble and the controlling of the production of SERS substrates by a simple measurement of the electrical conductivity leads to reproducible silver nanoparticles on a large scale. [8, 9]

\section{SERS and fluorescence readout}

To combine the advantages of fluorescence and SERS on the same plasmonic surface, gold nanorhomb arrays, allowing both the detection of fluorescence light and specific Raman fingerprint spectra, were used. The molecules of interest (here: label for DNA detection) are permit to bind at different distances from the metallic surface (see Fig. 1). The most efficient SERS enhancement is achieved for molecules in close vicinity (up to $5 \mathrm{~nm}$ ) to the metallic surface due to the strong field enhancement, while fluorescence is quenched most efficiently. Moreover, the fluorescence signal can be amplified for molecules with distances between 3 and $7 \mathrm{~nm}$ to the metal surface. The simultaneous application of SERS and fluorescence is demonstrated by the realization of a DNA detection scheme using the fluorescent dye Cy3.5 as label [10]. These investigations might be a contribution towards a more flexible application of the same biochip platform through the performance of both fluorescence and SERS microscopy.

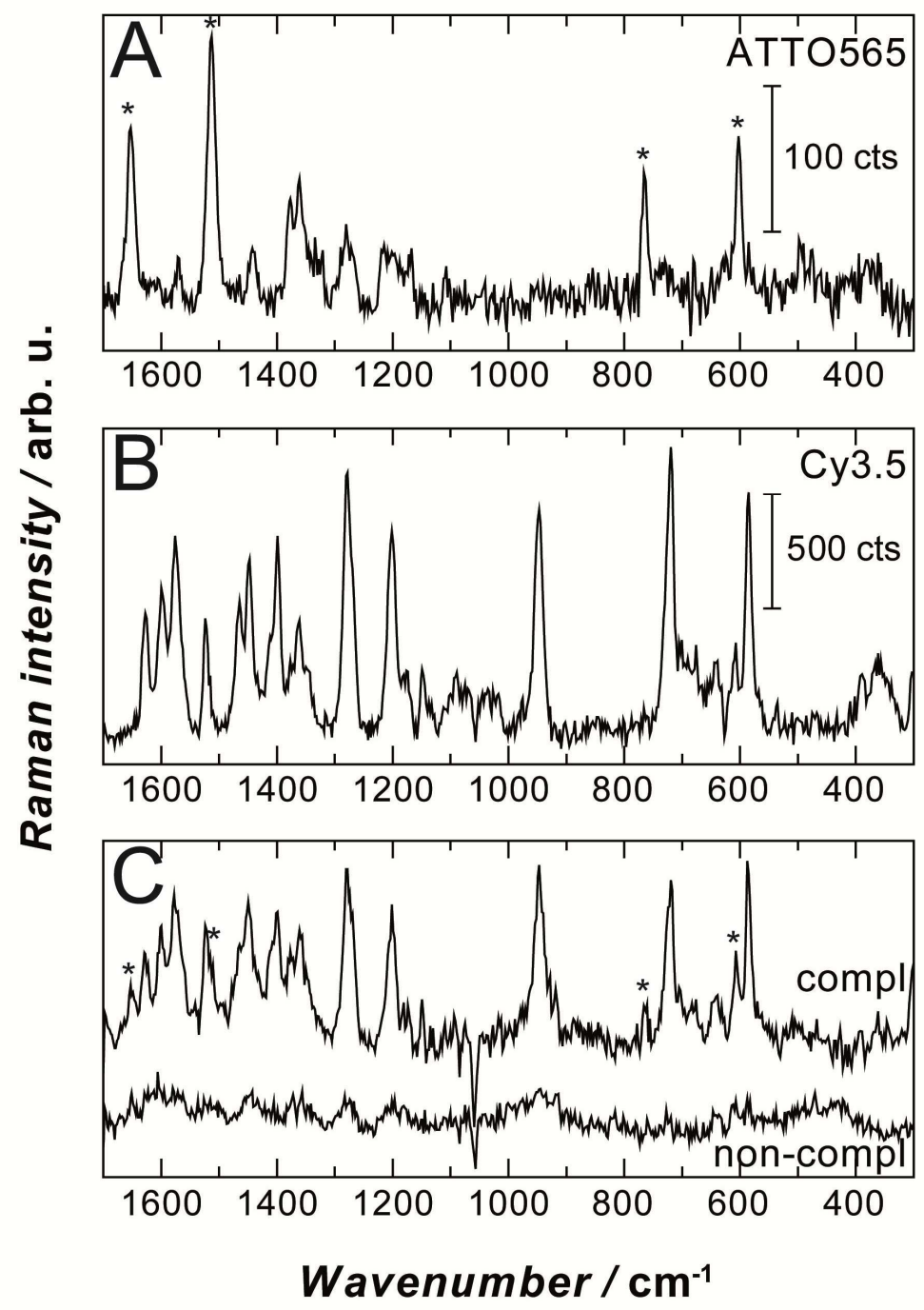

Fig. 2. The reference SERS spectra of the dye-labels Atto565 (A) and Cy3.5 (B) show significant different fingerprint signatures. (C) A multi-analyte approach has been applied by treating the modified sensor surface with a mixture of Atto565 and Cy3.5 labeled target DNA. The SERS signature for the complementary case is dominated by the contribution of the dye-labels. The Raman marker bands marked with an asterisk are assigned to contributions of Atto565. 


\section{Multi-analyte DNA detection scheme}

Since each molecule has a specific molecular Raman fingerprint spectrum, SERS is an attractive method to detect several molecules simultaneously [11]. Due to the fingerprint specificity the SERS technique overcomes the limited multiplexing capability of the fluorescence spectroscopy due to using fluorescent dyes with similar or overlapping emission spectra. In order to illustrate the simultaneous detection of dye labels with an almost same emission maximum, we have chosen Cy3.5 and Atto565 as reporter molecules. In Fig. $2 \mathrm{~A}$ and $2 \mathrm{~B}$ the reference SERS spectra are given for the dye labels. Since the dye spectra show clearly different signatures discrimination in a multi-analyte approach should be practicable. Thus, the biosensor was treated with a mixture of Atto565 and Cy3.5-labeled complementary target DNA. Representative multi-analyte SERS spectra for both the complementary and noncomplementary case are depicted in Fig. 2C. Since the Cy3.5 reference signature show more prominent Raman marker bands than Atto565, the multi-analyte fingerprint spectrum is dominated by the contribution of the Cy3.5 signature. The Raman marker bands marked with an asterisk are assigned to contributions of Atto565. Thus, both dye-labels are detected simultaneously opening a promising way towards a powerful multi-analyte DNA detection scheme by using regular ordered gold nanorhomb arrays as biosensor.

\section{Acknowledgements}

Funding of the research projects 'Photonic Nanomaterials (PhoNa)' within the framework 'Spitzenforschung und Innovation in den Neuen Ländern', furthermore 'QuantiSERS' and 'Jenaer Biochip Initiative' with the framework 'Unternehmen Region - InnoProfile Transfer' from the Federal Ministry of Education and Research, Germany (BMBF) is gratefully acknowledged.

\section{References}

[1] D. Cialla, A. März, R. Böhme, F. Theil, K. Weber, M. Schmitt, J. Popp, Surface-enhanced Raman spectroscopy (SERS): progress and trends, Analytical and Bioanalytical Chemistry 403, 27-54 (2012); doi: 10.1007/s00216-011-5631-x

[2] M. Porter, R. Lipert, L. Siperko, G. Wang, R. Narayanan, SERS as a bioassay platform: fundamentals, design, and applications, Chemical Society Reviews 37, 1001-1011 (2008); doi: 10.1039/b708461g

[3] U. Huebner, R. Boucher, H. Schneidewind, D. Cialla, J. Popp, Microfabricated SERS-arrays with sharp-edged metallic nanostructures, Microelectronic Engineering 85, 1792-1794 (2008); doi: 10.1016/j.mee.2008.05.005

[4] D. Cialla, R. Siebert, U. Hübner, R. Möller, H. Schneidewind, R. Mattheis, J. Petschulat, A. Tünnermann, T. Pertsch, B. Dietzek, J. Popp, Ultrafast plasmon dynamics and evanescent field distribution of reproducible surface-enhanced Raman-scattering substrates, Analytical and Bioanalytical Chemistry 394, 1811-1818 (2009); doi:10.1007/s00216-009-2749-1

[5] J. Petschulat, D. Cialla, N. Janunts, C. Rockstuhl, U. Huebner, R. Moeller, H. Schneidewind, R. Mattheis, J. Popp, A. Tuennermann, F. Lederer, T. Pertsch, Doubly resonant optical nanoantenna arrays for polarization resolved measurements of surface-enhanced Raman scattering, Optics Express 18, 4184-4197 (2010); doi:10.1364/OE.18.004184

[6] D. Cialla, J. Petschulat, U. Hübner, H. Schneidewind, M. Zeisberger, R. Mattheis, T. Pertsch, M. Schmitt, R. Möller, J. Popp, Investigation on the Second Part of the Electromagnetic SERS Enhancement and Resulting Fabrication Strategies of Anisotropic Plasmonic Arrays, ChemPhysChem 11, 19181924 (2010); doi:10.1002/cphc.200901009

[7] U. Huebner, K. Weber, D. Cialla, H. Schneidewind, M. Zeisberger, H. G. Meyer, J. Popp, Fabrication and characterization of silver deposited micro fabricated quartz arrays for surface enhanced Raman spectroscopy (SERS), Microelectronic Engineering 88, 1761-1763 (2011); doi:10.1016/j.mee.2010.12.097

[8] K. K. Hering, R. Möller, W. Fritzsche, J. Popp, Microarray-Based Detection of Dye-Labeled DNA by SERRS Using Particles Formed by Enzymatic Silver Deposition, ChemPhysChem 9, 867-872 (2008); doi:10.1002/cphc.200700591

[9] K. K. Strelau, T. Schüler, R. Möller, W. Fritzsche, J. Popp, Novel Bottom-Up SERS Substrates for Quantitative and Parallelized Analytics, ChemPhysChem 11, 394-398 (2010); doi:10.1002/cphc.200900867

[10] D. Cialla, K. Weber, R. Böhme, U. Hübner, H. Schneidewind, M. Zeisberger, R. Mattheis, R. Möller, J. Popp, Towards multiple readout application of plasmonic arrays, Beilstein Journal of Nanotechnology 2, 501-508 (2011); doi:10.3762/bjnano.2.54

[11] K. Faulds, R. Jarvis, W. E. Smith, D. Graham, R. Goodacre, Multiplexed detection of six labelled oligonucleotides using surface enhanced resonance Raman scattering (SERRS), The Analyst 133, 1505-1512 (2008), doi: $10.1039 / \mathrm{b} 800506 \mathrm{k}$ 